\title{
Les subordonnées asyndétiques en ancien français
}

\author{
Julie Glikman \\ Université Paris X Nanterre, Université de Potsdam, Modyco \\ julieglikman@yahoo.fr
}

\section{Introduction $^{1}$}

En ancien français, il existe des cas de propositions apparemment subordonnées non introduites par un marqueur de subordination, de type conjonction de subordination ou pronom relatif. Selon les terminologies, on parle alors de cas de parataxe, de subordonnées paratactiques ou de subordonnées asyndétiques (désormais SA). Les grammairiens signalent ce phénomène pour différents types de subordonnées. Ainsi, ils ont observé l'existence de SA complétives (Foulet 1928 : 333 ; Buridant 2000 : 571), de SA relatives (Ménard 1988: 78; Foulet 1928: 338; Buridant 2000: 575, 580), de SA « circonstancielles » exprimant la comparaison (Buridant $2000: 645$ ), l'hypothèse (voir Buridant, 2000 : 626,663 ) et la conséquence (Buridant, $2000: 619$ sq.). Ce phénomène, en outre, est considéré comme typique de l'ancien français et aurait disparu par la suite (Marchello-Nizia 1979, 1 : 428 ; Marchello-Nizia 1998 : 314). Bien que mentionné dans la plupart des grammaires de l'ancien français, ce phénomène ne semble cependant pas avoir fait l'objet d'études spécifiques décrivant de manière approfondie ses particularités, c'est pourquoi nous avons voulu étudier de plus près ce type de construction. Nous essayerons ainsi d'établir une description précise de ce phénomène, pour comprendre son fonctionnement et ses éventuelles particularités par rapport aux constructions introduites, et pouvoir ainsi, par la suite, tenter d'expliquer son évolution.

Nous avons procédé dans ce but au dépouillement d'une vingtaine d'œuvres du $11^{\mathrm{e}}$ au $14^{\mathrm{e}}$ siècle. Les œuvres sont de types divers, permettant ainsi un large échantillonnage (vers / prose ; chanson de geste / roman / récit historique / théâtre ; réécriture...). La liste des œuvres étudiées est présentée dans le tableau ci-dessous, les datations sont celles données par les éditeurs ou dans Soutet (1990: $45 s q q)$, les références complètes sont données en bibliographie :

\begin{tabular}{|l|l|l|l|l|}
\hline \multicolumn{1}{|c|}{ Euvre } & \multicolumn{1}{c|}{ Date } & \multicolumn{1}{c|}{ Vers/prose } & \multicolumn{1}{c|}{ Type } & Taille (lue) \\
\hline Eulalie & Peu apr. 881 & Vers & Hagiographie & $29 \mathrm{v}$ \\
\hline St Alexis & Milieu du XIe & Vers & Hagiographie & $625 \mathrm{v}$ \\
\hline Roland & 1100 & Vers & Ch. de geste & $4002 \mathrm{v}$ \\
\hline Louis C & $1131 ?$ & Vers & Ch. de geste & $2717 \mathrm{v}$ \\
\hline Louis AB & $1131 ?$ & Vers & Ch. de geste & $2671 \mathrm{v}$ \\
\hline Erec CdT & 1164 & Vers & Roman & $6879 \mathrm{v}$ \\
\hline Renart & Fin $12^{\mathrm{e}}$ & Vers & Parodie Ch. geste & $3256 \mathrm{v}$ \\
\hline Ste Marie T & Fin $12^{\mathrm{e}}$ & Vers & Hagiographie & $1532 \mathrm{v}$ \\
\hline Ste Marie X & Début $13^{\mathrm{e}}$ & Prose & Hagiographie & $14 \mathrm{p}$ \\
\hline St Nicolas & 1200 & Vers & Hagiographie & $1533 \mathrm{v}$ \\
\hline Clari & 1216 & Prose & Histoire & $106 \mathrm{p}$. \\
\hline Feuillé & 1277 & vers & Théâtre & $1098 \mathrm{v}$ \\
\hline Berthe & $13^{\mathrm{e}}$ & Vers & Ch. de geste & $3487 \mathrm{v}$ \\
\hline Rutebeuf & $13^{\mathrm{e}}$ & Vers & Poésie & $3138 \mathrm{v}$ \\
\hline Mort Artu & $13^{\mathrm{e}}$ & Prose & Roman & $266 \mathrm{p} / 79801 \mathrm{~g}$ \\
\hline Chartes & $13^{\mathrm{e}}$ & Prose & Chartes & $\sim 108 \lg$ \\
\hline Palatinus & Début $14^{\mathrm{e}}$ & Vers & Mystère & $1996 \mathrm{v}$ \\
\hline Saintré & 1455 & Prose & Roman/didactique & $\sim 300 \mathrm{lg}(100 \mathrm{p})$ \\
\hline Villon & 1461 & Vers & Poésie & $1360 \mathrm{v}$ \\
\hline Pathelin & 1465 & Vers & Farce & $1599 \mathrm{v} ?$ \\
\hline
\end{tabular}




\begin{tabular}{|l|l|l|l|l|}
\hline Pepin & $15^{\mathrm{e}}$ & Prose & Réécriture & $4475 \mathrm{lg}$ \\
\hline
\end{tabular}

Tableau 1: oeuvres du corpus

Le dépouillement manuel de ces œuvres a permis à l'heure actuelle de relever environ cent quatre-vingt dix cas de SA. On peut observer les tendances suivantes : les SA les plus fréquemment relevées sont les complétives, avec cent dix cas, viennent ensuite les consécutives, quarante cinq cas, puis les relatives avec trente et un cas, enfin nous avons également pu relever deux comparatives et deux hypothétiques. Cette répartition pourrait en partie s'expliquer par le fait que dans les cas des complétives par exemple, il est plus facile d'établir le lien de subordination, par la transitivité du verbe recteur entre autres, que dans le cas de subordonnées dites circonstancielles, où la différence entre deux indépendantes et deux propositions liées par un rapport de subordination n'est pas toujours facile à établir. Ceci pourrait expliquer l'absence dans le relevé d'autres types de subordination comme les temporelles, sans que cela signifie qu'il n'existe pas en ancien français de temporelles asyndétiques.

Pour essayer de mieux comprendre le phénomène des subordonnées asyndétiques et son fonctionnement, sa présence dans les textes, le fait qu'il soit donné comme disparu après cette période, nous avons essayé de voir quelle répartition générale s'observait dans notre corpus, ce qui a permis d'observer les tendances suivantes :

- dix cas proviennent d'œuvre en prose, cent quatre-vingt d'œuvre en vers ;

- en prose seul un cas est une relative, les autres sont toutes des complétives ;

- il semble que dans les textes en prose, les cas de SA sont plus en discours direct qu'en narration (3 seulement contre 7 en discours direct), alors qu'en vers la répartition discours direct / narration semble moins marquée (110 en discours direct contre 70 en narration);

- dans les textes en vers, la répartition des types de parataxes selon qu'il s'agit de narration ou de discours direct n'est apparemment pas la même : en discours direct, on a deux comparatives, sept relatives, une hypothétique, quinze consécutives et quatre-vingt six complétives, tandis qu'en narration on a une comparative, vingt-trois relatives, une hypothétique, vingt-neuf consécutives et seulement quinze complétives ;

- dans les complétives, la répartition en fonction de la personne du verbe recteur est la suivante: quarante-trois à la $1^{\text {ère }}$ personne, neuf à la $2^{\mathrm{e}}$, dix-neuf à la $3^{\mathrm{e}}$, deux à la $4^{\mathrm{e}}$, trente-deux à la $5^{\mathrm{e}}$ et trois à la $6^{\mathrm{e}}$.

La plus grande proportion de cas relevés dans les œuvres en vers peut aussi s'expliquer évidemment par le nombre plus important d'œuvres en vers dépouillées, mais, proportionnellement aux tailles et nombre des œuvres en vers et en prose, il ressort néanmoins que les cas de SA sont plus fréquents dans les textes en vers. Le fait qu'on observe plus de complétives dans les textes en prose n'est pas sans rappeler que les complétives sont, selon nous, comme nous l'avons déjà dit, le type le plus facilement identifiable. Il est également intéressant d'observer que dans les textes en prose, bien que le petit nombre d'exemples relevés ne permette pas de poser des affirmations trop tranchées, on trouve plus de SA en discours direct. En effet, la forte présence des SA dans les textes en vers a été souvent associé à la présence de la prosodie (Buridant 2000 : 569 ; Marchello-Nizia 1993), et donc, par la prosodie, à l'oralité.

Ces premiers chiffres semblent déjà pouvoir nous donner un aperçu de la représentativité et la répartition des SA dans les textes d'ancien français. Cependant, cela n'est pas encore suffisant pour expliquer cette répartition et bien cerner le fonctionnement des SA.

Pour mieux comprendre ce phénomène, il faut donc encore procéder à une analyse plus fine de son comportement. Il faut pour cela le mettre également en perspective avec la langue de l'œuvre dans laquelle il se trouve, pour comprendre comment les SA s'intègrent et fonctionnent dans le système de la langue. Nous avons pour cela choisi d'approfondir ici l'analyse des SA dans une seule œuvre, à savoir Le Roman de Renart. Il nous a paru intéressant de le retenir comme corpus de départ dans le cadre de cette étude, car il offre un grand nombre d'exemples de SA. 
Dans un premier temps, nous interrogerons la nature du lien reliant ces couples de propositions pour établir si l'on peut parler à juste titre de subordination. Nous essaierons ensuite de faire ressortir les particularités et caractéristiques des SA dans le texte.

\section{Sur le statut des SA}

\subsection{Observations}

Dans ce type de construction, on se trouve en présence de deux constructions verbales juxtaposées. L'absence de morphème de liaison, de type subordonnant ou coordonnant, fait qu'il est difficile de statuer sur la nature du lien qui les unit. Cependant, les deux propositions ne semblent pas être indépendantes l'une de l'autre. Il semblerait plutôt qu'elles constituent une même unité supérieure d'ordre micro syntaxique, ou une même phrase au sens traditionnel du terme, entretenant une relation de subordination entre elles. Cela apparaît d'autant plus clairement quand on met en parallèle des exemples équivalents qui, eux, comportent une conjonction de subordination ou un pronom relatif, comme dans les couples (1)(2), (3)-(4) et (5)-(6) :

(1) Si tot com Renart entre a cort, / il n'i a beste ne s'atort / ou de clamer ou de respondre. (122123)

[Aussitôt que Renart entre à la cour, il n'y a de bête [qui] ne s'apprête ou à se plaindre ou à prendre sa défense $]^{2}$

(2) Onques n'i ot beste si ose / qui se tardast por nule chose, (19-20)

[jamais il n'y eut de bête si hardie qui ne se mette en retard pour aucune raison]

(3) Mes or est Renart pris au laz / et set bien ce n'est mie a gas ; / bien set ne se puet or desfandre (1361-63)

[Mais à présent Renart est pris au collet et sait bien [que] ce n'est pas une plaisanterie ; [il] sait bien [qu'] à présent [il] ne peut pas se défendre]

(4) Renart li rous fremist et tranble : / bien set que sa mort est juree, (1350-51)

[Renart le roux frémit et tremble : [il] sait bien qu'on a juré sa mort]

(5) de chascun li fis tant mengier / n'en pot issir, si fu emflez, (1070-71)

[de chacun [je] lui fis tant manger [qu'il] n'en put sortir, tellement [il] était enflé]

(6) tant a sachié que a grant paines / estant les piaus, ronpent les vaines (675-76)

[[il] a tant tiré qu'avec beaucoup de douleur il distend sa peau, les veines rompent]

Ainsi, en (1), (3) et (5) on a une construction asyndétique à laquelle correspondraient les variantes introduites de (2), (4) et (6). On observe également, comme signalé précédemment, que les SA peuvent apparaître dans différents types de propositions subordonnées : complétives, relatives, consécutives, voire dans le système hypothétique.

La question est de savoir si l'on peut vraiment parler de subordination dans les cas non introduits, et, dans l'affirmative, quels sont les critères pouvant justifier ce choix.

\subsection{Quelques critères pouvant servir à établir le lien de subordination}

Le parallèle avec des formes introduites peut servir d'indice. Toutefois, il ne nous semble pas suffire à lui seul pour établir le lien de subordination entre les deux propositions. Nous présenterons ici quelques critères supplémentaires. 
Dans certains cas, la seconde proposition vient saturer une place de valence ouverte par le verbe de la première construction. Elle doit donc être analysée en tant que SA, faute de quoi il y aurait un argument manquant dans le premier énoncé, comme en (7) avec gardez :

(7) gardez sanz lui ne retornez. (950)

[Veillez [à ce que vous] ne reveniez pas sans lui]

Dans d'autres cas, la présence du subjonctif, mode de la subordination, dans la SA peut aussi servir de critère, ainsi que la présence d'un terme corrélatif dans les constructions corrélées, qui, bien que n'étant pas en soi un critère de dépendance, montre qu'une autre proposition est attendue (voir en (5) tant).

L'analyse comme SA est parfois la seule analyse possible, comme en (1) où la partie en italique doit être analysée comme une relative asyndétique : le mot beste est en facteur commun entre la relative, dont il représente virtuellement le sujet (à travers le pronom relatif sujet non exprimé) et l'énoncé de rang supérieur (où le groupe beste + relative forme le complément de il n'i a). De plus, après il n'i a bestes, il faut forcément une proposition relative (relative dite déterminative dans ce cas) pour compléter bestes sinon la phrase ne fait pas sens.

Les grammairiens de l'ancien français ont observé cette possibilité de construction de la phrase en ancien français : «quand le caractère subordonné de la proposition qu'il introduit est suffisamment signifié par d'autres moyens, le subordonnant peut être effacé » (Buridant $2000: 571$ ).

Cependant, il reste parfois des occurrences où il demeure difficile d'établir s'il s'agit vraiment d'un lien de subordination. Cela peut être le cas en l'absence de tout indice supplémentaire, lorsque la SA n'occupe pas la place d'un complément obligatoire, ou dans les cas de verbes recteurs pouvant avoir une double construction, avec complétive régie ou en construction absolue. Le problème semble se poser d'autant plus avec une catégorie de verbes dits recteurs faibles (Blanche-Benveniste 1989) ou verbes parenthétiques (Andersen 1996, voir aussi Schneider 2007). En effet, ces verbes peuvent parfois régir une complétive, mais peuvent aussi être intégrés (anté-/ inter-/ ou postposés) à une autre prédication qu'ils ne régissent pas, mais à laquelle ils sont incidents ${ }^{3}$. Il faut parfois regarder de plus près l'ensemble des occurrences du verbe dans le texte pour mieux comprendre son fonctionnement, comme dans l'exemple ci-dessous :

(8) Sachiez, se vos le me donez, / bien vos sera guerredonnez (1509-10)

[Sachez [que], si vous me le donnez, [cela] vous sera bien rendu]

(9) Veez le la ou il s'enfuit ; / sachiez bien, se il nos estort, / nos somes tuit jugié a mort, (160608)

[Voyez le là où il s'enfuit; sachez bien [que], s’il nous échappe, nous sommes tous condamnés à mort]

(10) S'il est panduz, sachiez de fi, / tuit si parent ierent honi, (1443-44)

[S'il est pendu, sachez assurément, tous ses parents seront honnis]

Dans ces exemples on trouve le verbe savoir à la cinquième personne de l'impératif. Andersen (1996) rapproche ce type d'emploi des emplois parenthétiques, donc non recteurs. Cependant, si l'on regarde l'ensemble des occurrences de sachiez, on arrive à établir deux fonctionnements distincts entre rection et incidence. Il semble qu'on puisse opérer une distinction selon la place du verbe savoir par rapport à l'hypothétique présente dans ces exemples.

Dans un premier cas de figure (exemple (10)), «sachiez de fi » se trouve inséré entre la protase et l'apodose. Le fait qu'il soit inséré donne à penser qu'il est clairement en emploi parenthétique, ce que semble confirmer l'exemple (11) :

(11) Par toz les sainz de Belleant, / se mes en oi ne tant ne quant, / sachiez le bien, sanz demorance / panduz sera a une branche. " (1465-68) 
[Par tous les saints de Bethléem, si dorénavant [j']en entends parler en quoi que ce soit, sachez le bien, sans délai, [il] sera pendu à une branche]

La présence d'un pronom dans «sachiez le bien» peut être une preuve de la possibilité d'un emploi autonome, c'est-à-dire non rattaché syntaxiquement au reste de la phrase. Ceci semble enfin être confirmé par le fait qu'il n'existe aucun emploi de ce type, avec sachiez inséré entre la protase et l'apodose, avec la conjonction que.

Les exemples (8) et (9) paraissent fonctionner différemment : sachiez se trouve en tête d'énoncé, et non pas en insertion, et il n'existe pas dans le texte d'exemple de ce type avec un complément pronominal. Au contraire on trouve un exemple équivalent construit avec la conjonction que, donc avec une complétive introduite (exemple (12)).

(12) bien sachiez que, se Renart vit, / tel le conperra q'ainz nel vit. (1769-70)

[sachez bien que, si Renart vit, celui-là le payera cher qui ne l'a jamais vu]

On peut penser que la présence de se en (8) et (9) facilite la parataxe, suffisant à marquer les frontières entre propositions tout en évitant la succession de deux conjonctions.

Il semble donc qu'on puisse parfois trouver des indices permettant d'établir s'il y a rection ou non. Mais, malgré notre tentative de trouver des critères formels permettant d'établir s'il y a ou non rection, cela ne suffit pas toujours. Il y a des cas où seule la présence d'un parallèle comprenant une variante introduite (par une conjonction ou un pronom), ou la restitution du sens, permet de penser qu'il s'agit bien de subordination. Dans certains cas, il reste toujours impossible de trancher entre analyse en tant que proposition matrice - subordonnée ou propositions indépendantes ${ }^{4}$.

\section{Caractéristiques et particularités des SA dans le Roman de Renart}

Dans cette partie, nous allons nous intéresser aux caractéristiques des SA dans Le Roman de Renart. En effet, nous venons de voir qu'il est possible d'établir que les SA sont bien des subordonnées au même titre que les subordonnées introduites. En conséquence, la question qui se pose maintenant est la suivante : existe-t-il des caractéristiques particulières aux SA qui les différencieraient des subordonnées introduites ? Y a-t-il des contextes d'emploi particuliers, des blocages au niveau des constructions possibles? Nous regarderons pour répondre à cette question les caractéristiques des SA pour chaque type observable, dans les propositions relatives, complétives, dans les constructions corrélées et dans le système hypothétique.

\subsection{Propositions subordonnées relatives}

La possibilité de construction de la proposition relative en parataxe a été observée par les grammairiens de l'ancien français : «Le relatif absent est d'ordinaire le sujet qui, très rarement le régime que ou cui » (Ménard $1988: 78$ ) ; « [Le relatif] peut ne pas être exprimé, en corrélation avec tel [...] dans l'expression négative de la totalité $[\ldots]$ en particulier dans l'expression $n$ 'i a celui » (Buridant $2000: 580$ )

Dans notre texte, on relève trois cas de relative non introduite :

(13) Quant a la cort vint la novele, / a tex i ot ne fu pas bele; (487-88)

[Quand la nouvelle vint à la cour, il y en eut [à qui] [elle] ne fut pas agréable]

(14) Si tot com Renart entre a cort, / il n'i a beste ne s'atort / ou de clamer ou de respondre. (122123)

[Aussitôt que Renart entre à la cour, il n'y a de bête [qui] ne s'apprête ou à se plaindre ou à prendre sa défense]

(15) N'i a celui n'aie fait honte; (1764) 
Comme le signalaient les auteurs ci-dessus, on retrouve en effet dans nos exemples le fait que le relatif non exprimé serait en position sujet dans la relative, en (14), mais ce n'est pas le cas en (13) et (15), ce qui montre qu'une autre position est également possible. On observe également la corrélation avec tel ou celui, qui rend seule possible l'analyse en relative ((13), (15)) et la négation de la totalité ((14), (15)), avec dans ces deux cas le subjonctif dans la relative, marquant le virtuel. Il semble donc que les cas de SA dans les relatives n'apparaissent que dans des conditions bien précises et assez marquées. Cependant, ceci pourrait également s'expliquer par le fait que c'est justement ce contexte très marqué qui permet d'identifier la relative asyndétique. En effet, dans les cas de ce genre, l'analyse comme relative asyndétique est la seule possible et ne permet pas de doute. A l'inverse, en dehors de ce type de contexte, il n'est pas toujours possible de faire la différence entre une simple juxtaposition de propositions et une proposition relative, comme dans l'exemple suivant :

(16) Adont si alerent tant qu'il vinrent a une chité, Poles avoit a non. (Clari, p.62, XIII, 35-36)

[Ils naviguèrent alors tant et si bien qu'ils arrivèrent à une cité du nom de Pola] (Trad. J. Dufournet)

Bien que le traducteur ait traduit « Poles avoit a non » par un complément du nom, donc lié, il n'y a aucun indice syntaxique en ancien français pour une telle analyse, l'expression du pronom sujet n'étant pas obligatoire, son absence ici ne peut pas servir d'argument. On pourrait donc y voir tout aussi bien une relative [qui s'appelait Pola] qu'une indépendante [elle s'appelait Pola].

\subsection{Propositions subordonnées complétives}

Les grammaires signalent qu'en ancien français il est possible de trouver des propositions complétives non conjonctives après des verbes de volition, d'ordre, de prière, de promesse, d'opinion, de connaissance (Buridant $2000: 571$; Foulet $1928: 333$ ). Dans notre texte, on peut effectivement trouver des SA régies par :

- des verbes d'opinion :

savoir (cinq occurrences) :

(17) Renart set bien ce est li ors : (513)

[Renart sait bien [que] c'est l'ours]

cuidier (sept occurrences) :

(18) ce cuit vos n'en gouterïez. (823)

[je (le) crois [que] vous n'en goûteriez pas]

penser (une occurrence) :

(19) Quant l'ors ot des vilains la rage, / fremist et panse en son corage / miauz li vient il le musel perdre, / einz que Lainfroiz le puisse aerdre (669-72)

[Quand l'ours entend la rage des paysans, [il] frémit et pense en lui-même [qu']il lui vaut mieux perdre le museau plutôt que Lainfroiz puisse l'attraper]

vouloir (une occurrence) :

(20) Se mon seignor le roi plesoit / et une chose me faissoit / que il vousist je fusse moines, / reclus, hermites ou chanoines, [...] (2067-70)

[S'il plaisait à mon seigneur le roi et s'il m'accordait que il voulût bien [que] je devinsse moine, reclus, ermite ou chanoine...] 
- des verbes d'empêchement et de crainte :

garder (deux occurrences) :

(21) gardez sanz lui ne retornez. (950)

[veillez [à ce que vous] ne reveniez pas sans lui]

garir (une occurrence) :

(22) ja li vostres coarz chevaux / ne vos guerra de mes cheaux / ne lor en face livroison. (1547-49)

[jamais votre cheval couard ne vous protègera pas [que] je vous livre en pâture à mes petits]

criembre (une occurrence) :

(23) mes je sant moines a si fax / que je criem ne me mesavaingne, / se ge faz tant moignes devainne. (1032-34)

[mais je sens les moines si faux que je crains [qu'il] ne m'arrive malheur, si je fais en sorte [que] je devienne moine]

- des verbes de déclaration à valeur jussive :

dire (une occurrence) :

(24) Dites de par moi le reçoive, (1987)

[Dites [qu'il] le reçoive de ma part]

mander (une occurrence) :

(25) Mandez Renart de moie part / atandu l'ai .III. jorz antiers. (462-63)

[Faites savoir à Renart de ma part [que je] l'ai attendu trois jours entiers]

estre seür (une occurrence, à l'impératif) :

(26) Passez outre: se vos chaez, / saiez seür vos floterez. (1381-82)

[Passez de l'autre côté : si vous tombez, soyez sûr [que] vous flotterez]

Nous observons donc que les SA complétives sont possibles après une large palette de verbes. De plus, les verbes recteurs de complétive les plus fréquents dans l'œuvre (relevé établi sur un échantillon des 1500 premiers vers : dire, savoir et cuidier) acceptent tous la construction avec SA.

Dans dix cas sur vingt-trois, on observe la présence du subjonctif dans la SA (voir aussi (18), (20), (22) (23), (24), (31), (32), (33) et les vers 1077-78) :

(27) [...] et Roomiax qui le tesmoigne, / la cort cuidast ce fust mançonge. (485-86)

[...et Roonnel qui en témoigne, la cour aurait cru [que] c'était un mensonge]

Avec cuidier, tous les cas ont le subjonctif dans la subordonnée, sauf un cas à l'indicatif :

(28) Je quit Renart vos a loé / et de son miel vos a doné, (1391-92)

[Je crois [que] Renart vous a loué et vous a donné de son miel]

L'indicatif est ici présent pour insister sur le degré de certitude du locuteur. Il est intéressant par ailleurs de constater qu'avec ce verbe on trouve plus de SA que de complétives introduites (sept cas contre cinq). En effet, la présence du subjonctif dans la subordonnée, mode le plus couramment employé avec ce verbe (dix cas sur douze dans le texte), peut constituer un marqueur suffisant de subordination (Buridant, 2000 : 571), ce qui pourrait expliquer le nombre élevé de SA après ce verbe. 
Le verbe recteur peut être à l'indicatif à la première (cinq cas, voir (18), (23), (28), (32) et (33)), troisième (sept cas : (17), (19), (20), (22), (27), (34), voir aussi vers 1077-78) ou cinquième personne ((26), voir aussi vers 212), à différents temps (par exemple au présent, dans (18), comme au passé simple dans (27)). Le verbe recteur peut également être à la cinquième personne de l'impératif ((21), (24), (25), (29), (31), (35), (36)) ; on note également la présence de l'impératif dans la subordonnée en (21) et (29) (voir à ce sujet Buridant, 2000 :734-35). On observe donc qu'il existe là aussi un large éventail de possibilités en ce qui concerne le mode et le temps dans les constructions contenant une SA.

Dans le cas de garder cependant, on observe que dans les occurrences relevées garder est toujours à l'impératif :

(29) Gardez nel dites a nului, (1990)

[Veillez [à ce que vous] ne le disiez à personne]

On pourrait en conclure qu'il s'agit d'une particularité liée à la construction avec SA. Cependant, si l'on regarde de plus près l'ensemble des occurrences de garder suivies d'une complétive dans le texte, on constate que dans les cas non asyndétiques le verbe est également à l'impératif, ce qui laisserait à penser que la présence de l'impératif pour le verbe recteur n'est pas une particularité liée à la construction avec complétive asyndétique, mais à la construction du verbe lui-même :

(30) Gardez que vos n'en parlez plus. (2745)

[Veillez [à ce que] vous n'en parliez pas davantage]

Les exemples (17), (19), (27) et (34) relèvent de la narration, tandis que les autres, donc une grande majorité, relèvent du discours direct.

On peut également observer la négation du verbe recteur comme ci-dessous (voir aussi en (22)) :

(31) ne quidez mie je vos hace. (803)

[ne croyez pas [que] je vous haïsse]

(32) ne cuit devant un an vos faille. (1140)

[[je] ne crois pas [qu'il] vous [en] fasse défaut avant un an]

(33) ne cuit devant un an me faille: (1786)

[[je] ne crois pas [qu'il] m'[en] fasse défaut avant un an]

Ainsi que la négation dans la SA en (18), (21), (22), (29) et ci-dessous en (34), la présence de ne pouvant suffire à marquer les limites entre proposition (également en (23) avec un ne explétif) :

(34) Mes or est Renart pris au laz / et set bien ce n'est mie a gas ; / bien set ne se puet or desfandre (1361-63)

[Mais à présent Renart est pris au collet et sait bien [que] ce n'est pas une plaisanterie ; [il] sait bien [qu'] à présent [il] ne peut pas se défendre]

On a aussi parfois l'insertion d'une proposition entre la proposition rectrice et la proposition régie ${ }^{5}$ :

(35) Sachiez, se vos le me donez, / bien vos sera guerredonnez (1509-10)

[Sachez [que], si vous me le donnez, [cela] vous sera bien rendu]

(36) Veez le la ou il s'enfuit; / sachiez bien, se il nos estort, / nos somes tuit jugié a mort, (160608)

[Voyez le là où il s'enfuit; sachez bien [que], s'il nous échappe, nous sommes tous condamnés à mort] 
Une construction clivée en «ce fu » construite de manière asyndétique a également pu être relevée (bien que dans ce cas il ne s'agisse pas à proprement parler de complétive), également avec insertion d'une proposition :

(37) La riens qui plus le desconforte / ce fu, qant il vint a la porte, / entre le fraisne et le sapin / a veü l'oisel saint Martin; (769-72)

[la chose qui l'inquiète le plus, ce fut [que], quand il vint à la porte, entre le frêne et le sapin, [il] a vu l'oiseau de Saint Martin]

Nous pouvons donc constater qu'il existe diverses possibilités de construction pour les SA complétives, tant au niveau des temps et modes, des personnes, de la présence de la négation. Il ne semble donc pas qu'il y ait de restriction particulière dans leur emploi. De plus, quand il semble y en avoir, comme dans le cas de garder, à l'impératif, nous avons pu établir qu'il ne s'agit pas d'une particularité liée à la présence de la SA, mais au verbe lui-même.

\section{3 Éléments corrélateurs de consécutives : tant, tel, si, a pou}

Ces éléments peuvent constituer des termes corrélatifs, avec que, comme termes d'appel pour exprimer la conséquence. Ces consécutives peuvent également être construites en parataxe, par «ellipse de que » (Buridant, $2000: 619$ sq.), la présence d'un seul terme corrélatif pouvant suffire à appeler la deuxième proposition.

\subsubsection{Tant}

La moitié des occurrences de tant dans le texte est employée comme élément corrélateur appelant une consécutive (la construction en $a$ tant constituant pour sa part un quart des emplois, comme articulateur de récit: voir à ce sujet Buridant, $2000: 175)$ et marquant «la conséquence d'un procès mené à un certain degré de quantité » (ibid. : 621).

Sur cette moitié d'usage en tant que corrélateur de consécutive, environ un tiers des occurrences est construit en parataxe et se trouve en grande majorité dans des passages de discours direct, comme dans les exemples ci-dessous, où la négation dans la consécutive peut «[faire] office de corrélation avec le strument d'appel » (ibid. : 622) (environ la moitié des cas en parataxe, contre un seul cas avec négation dans la consécutive dans les cas introduits), et avec une double corrélation avec si en (39) :

(38) de chascun li fis tant mengier / n'en pot issir, si fu emflez, (1070-71)

[de chacun [je] lui fis tant manger [qu'il] n'en put sortir, tellement [il] était enflé]

(39) Renart est si vers moi mesfaiz, / tant a anuiz a trestoz faiz, / nus ne le vos porroit retraire, (2125-27)

[Renart est envers moi si coupable, [il] a fait tant d'ennuis à tous, [que] nul ne pourrait vous le rendre]

Voir aussi (23) et vers 187, 276, 697, 952, 1454, 1583, 1794, 1933, 2037.

On trouve également un cas d'inversion : «consécutives interverties, la conséquence étant exprimée en protase avant l'apodose en tant $\gg$ (ibid. : 623) :

(40) [...] et sire Nobles li lions, / s'eüst o lui toz ses barons, / nou trairoient de la tainiere, / tant est la terre fort et fiere. (1357-60)

[...et sire Noble le lion, s'[il] eût avec lui tous ses barons, [ils] ne le sortiraient pas de la tanière, tant la terre est forte et fière]

On pourrait penser en l'absence de que qu'il s'agit parfois non pas de marqueur de consécution, mais d'un tant intensif. Cependant, Weill (2002) a bien montré qu'il ne faut pas penser en terme d'intensif, les 
emplois de tant comme intensif étant bien moins fréquents en ancien français qu'en français moderne. Elle a au contraire mis au jour des emplois de tant comme marqueur de transfert, de type « de telle sorte que » :

\begin{abstract}
L'interprétation traditionnelle de tant intensif en phrase exclamative me paraît fausser le sens des textes. [...] Je pense que considérer tant comme un marqueur de consécution, de transfert fonctionnant de façon efficace permettra une autre approche de certains passages. (Weill, $2002: 406$ )
\end{abstract}

Il paraît donc légitime de continuer de traiter ces exemples comme des cas de SA. Nous avons pu voir ici que ces cas de SA représentent une part importante de l'ensemble des constructions consécutives avec tant, et on peut également observer leur forte présence en discours direct.

\title{
3.3.2 Tel
}

Un tiers des emplois de tel comme élément corrélateur d'une consécutive sont en parataxe ${ }^{6}$ :

(41) Isangrin a bien tel puissance, / se Renart pres de lui mannoit / et por la pes ne remannoit / qui novelement est juree, / ja envers lui n' avroit duree. (60-64)

[Isangrin a bien une telle puissance [que], si Renart était près de lui et ne restait tranquille pour la paix qui est nouvellement jurée, il n'aurait jamais de patience envers lui]

(42) mais, ainz c'om doie vandangier, / cui ge Renart mener tel guerre / ne le guerra ne ciel ne terre / ne murs ne fossez desfansables. (266-69)

[mais, avant qu'on doive vendanger, je crois mener à Renart une telle guerre [que] ne le protègera ni ciel ni terre ni mur ni fossés défendables]

(43) je vos fis ja faire tel cors, / qant vosites le miel mengier ; / bien me cuidai de vos vangier ; $(1722-24)$

[je vous fis alors faire une telle course quand vous vouliez manger le miel [que] je crus bien me venger de vous]

On peut observer dans ces exemples l'insertion d'une autre proposition introduite entre la protase et l'apodose : l'hypothétique «se [...] juree» en (41) et la temporelle «qant vosites le miel mengier» en (43), et la présence de la négation dans la consécutive en (42).

\subsubsection{Si}

On peut dégager dans le texte principalement trois constructions possibles de $s i$ :

- comme articulateur de discours (environ $59 \%$ des cas, voir Buridant, $2000: 505$ sq.), toujours placé en tête de proposition devant le noyau verbal, composé du verbe et éventuellement de ses clitiques, voire d'un adverbe :

(44) et il se traist mout tost ariere, / si se feri en sa taisniere. (43-44)

[et il se tire bien vite en arrière, et se cache dans sa tanière]

- dans la construction si com (environ $10 \%$, voir ibid. : 638 « la comparaison hypothétique »);

- et enfin comme corrélateur de consécutive dans la locution si que, laquelle peut être disjointe (ibid. : 620) (environ $27 \%$ des occurrences). Parmi ces $27 \%$ dans cet emploi, $37 \%$ présente la non expression du que (voir aussi exemple (29) «Renart est si vers moi mesfaiz », avec tant coordonné) ${ }^{7}$ :

(45) eüst ma fame si baillie / contre son cuer l'eüst saisie, (93-94)

[... eût traité ma femme de telle sorte [qu'il] l'eût prise contre sa volonté...] 
(46) Diex gart au provoire tel fil / qui aprant si bien a barat / panre quide gorpil et chat! (864-66) [que Dieu évite au prêtre un tel fils qui apprend si bien la ruse [qu'il] croit prendre renard et chat!]

(47) et si le vont si esplumant, / si li poilent son peliçon / en haut en volent li flocon. (1646-48) [et [ils] vont en le plumant si bien, [ils] lui pèlent tant le pelage [que] les flocons en volent haut]

(48) mout par est forz vostre donjons, / mes n'est si forz ne l'aie pris; (1772-73)

[votre donjon est très fort, mais [il] n'est si fort [que] [je] ne l'aie pris]

(49) Cist chastiaus est si bien asis / ja par force ne sera pris. (1797-98)

[Ce château est si bien assis [que] [il] ne sera jamais pris par force]

(50) dou deslïer est si hastez / assez i a des antamez; (1880)

[[il] est si pressé de détacher [que] [il] y [en] a assez d'entamé]

(51) entre ses danz si fort l'estraint / morir le fait, si le destraint ; (1957-58)

[[il] le serre si fort entre ses dents [qu'il] le fait mourir, alors [il] le relâche]

On peut en général assez bien faire la distinction entre l'emploi comme cheville discursive et l'emploi comme élément corrélateur de consécutive par la structure même, la construction comme articulateur de discours étant du type $[s i+(\mathrm{adv}$.) (clitique) V] tandis que les emplois de $s i$ corrélateur de consécutives se font généralement selon le schéma [V + si (bien) + Adj. / Nom / Participe + (que) P]. On remarquera par ailleurs que la construction en être + attribut est très présente $((48),(49),(50))$. On observe également la présence de la négation dans la consécutive, en (48) avec emploi du subjonctif, pouvant marquer « une conséquence à réaliser » (Buridant, 2000 : 620) et en (49) appuyé par ja. En (47), le pronom de rappel en dans la consécutive « en volent » rappelle la protase et fait ressortir la notion de conséquence.

L'exemple suivant, bien que considéré par l'éditeur, vu la ponctuation, comme une construction de deux propositions indépendantes, nous paraît pouvoir également être analysé comme une consécutive corrélée au même titre que celles ci-dessus :

(52) " Aï, fait il, rous de pute aire, tant par ies or de mal afaire et si es male criasture. Certes en toi pecha Nature, qant tu ne puez a bien entendre, filz a putain, dines de pandre. " (1453-58)

[Ah, fait-il, sale rouquin, [tu] as une si mauvaise nature et [tu] es si mauvaise créature [que] certes la nature pêcha en toi, quand tu ne peux pas vouloir le bien, fils de pute, digne d'être pendu]

La proposition commençant par certes constituerait la proposition consécutive doublement appelée en protase par tant et $s i$; en effet, bien que dans ce cas les éléments corrélateurs soient antéposés au verbe estre, on retrouve la construction typique V être + attribut.

\subsubsection{A pou / a poi}

Buridant parle pour ces types de constructions en a pou / a poi (que) de "conséquence imminentielle » (Buridant, $2000: 622$ ), dans lesquelles la consécutive est niée et l'ellipse de que possible. On peut en effet signaler que dans le texte, sur les six occurrences de ce type, trois présentent l'absence de que ${ }^{8}$ :

(53) et sache et tire et riens ne vaut ; / a pou la qeue n'est rompue, (1868-69)

[et tire et tire en vain, peu s'en faut que la queue n'est rompue]

(54) Ja li pertuisoient les rains, / a poi ne chiet entre lor mains ; (1649-50) 
[Déjà ils lui piquent les reins, peu s'en faut qu'il ne tombe entre leurs mains]

(55) Et li autre sachent et tirent, / a poi li cul ne lor descirent. (1871-72)

[et les autres tirent et tirent, peu s'en faut qu'ils ne leur déchirent le cul]

\subsection{Le système hypothétique}

En ancien français, le système hypothétique, ou hypothético-concessif, peut s'exprimer au subjonctif, avec verbe en tête (voir Buridant, $2000: 626,663$ ). Dans le texte, il semble qu'on puisse trouver un exemple de ce type de système, en (56) :

(56) Et je sai bien, que que nus die, / que cil qui tot le mont conchie, / Renart, icil mavés lechierres, / cil rous puanz, cil orz trichierres, / eüst ma fame si baillie / contre son cuer l'eüst saisie, / ja Maupertuis nel garentist, / ne forteresce qu'il feïst / que je ne l'eüse tué / et puis en un conpaing rué (89-98)

[Et je sais bien, quoi que l'on dise, que [si] celui qui trompe tout le monde, Renart, ce mauvais larron, ce rouquin puant, ce tricheur, avait traité ma femme de telle sorte [qu'il] l'eût prise contre son cœur, jamais Maupertuis ne l'aurait protégé ni aucune forteresse qu'il eût fait que je ne l'aie tué et ne l'aie précipité en un bourbier.]

On aurait en protase « cil qui [...] saisie » et en apodose « ja Maupertuis [...] rué ». Même si la protase ne présente pas l'ordre V- Sujet, habituel dans la protase asyndétique (cf. en FM : «Fût-ce... »), la présence d'un système subjonctif plus-que-parfait (eüst baillie)-subjonctif imparfait (garentist) conforte bien cette lecture. De plus, eüst est placé en tête de vers, ce qui produit tout de même un effet marqué du même type que l'effet V1. Le fait que le système hypothétique soit ici lui-même enchâssé dans une proposition complétive introduite régie par je sai que a pu conduire à une expression paratactique du système hypothétique, évitant ainsi la présence de deux conjonctions à la suite et explique peut-être également le fait que le verbe ne se trouve pas en tête de la protase, fait qu'on pourrait également mettre en relation avec la taille importante de cette protase.

\section{CONCLUSION}

Nous avons pu montrer qu'il existe des constructions asyndétiques dans Le Roman de Renart, environ une soixantaine de cas sur les deux mille deux cent soixante vers étudiés. Ces cas de parataxes peuvent relever de différents types de subordination: propositions relatives, complétives, consécutives et hypothétiques. Un certain nombre de critères peuvent leur être associés, voire aider à leur reconnaissance (voir aussi à ce sujet Glikman à par. a et b) : présence du subjonctif, d'un élément corrélatif, de la négation dans la subordonnée, insertion d'une proposition entre la proposition rectrice et la subordonnée, type de verbe recteur de SA; sur ce dernier point, on peut en effet trouver, dans le texte, des SA après certains types de verbes, appartenant aux catégories des verbes d'opinion, de parole, de connaissance, de volition, d'empêchement, certains de ces verbes étant parfois même majoritairement construits en parataxe dans le texte (voir cuidier). Enfin, on peut également signaler que plus des deux tiers des cas de parataxe dans le texte se trouvent dans du discours direct, alors que la répartition discours direct - narration est à peu près égale pour l'ensemble du texte, ce qui semblerait confirmer certaines théories avançant que la parataxe relèverait plus de l'oralité.

Nous pouvons donc constater que cette analyse des SA dans Le Roman de Renart nous a permis de confirmer les tendances observées en introduction sur un corpus plus large. Mais surtout, cela nous a permis de mettre en perspective ces tendances avec le fonctionnement général du texte. En effet, par cette analyse nous pouvons montrer si une tendance est vraiment liée à la construction asyndétique, comme nous venons de le signaler pour la répartition entre discours direct et narration, qui montre bien que l'importance des SA en discours direct n'est pas uniquement liée au fait qu'il y aurait plus de discours direct dans le texte, mais bien au fait que cette construction est plus présente dans le discours direct. Cela 
nous permet également, à l'inverse, de montrer quand une tendance n'est en fait pas spécifique de la construction avec SA, comme dans le cas de garder qui apparaît toujours à l'impératif dans les cas relevés, mais est en fait dans l'œuvre toujours à l'impératif quand il régit une complétive, qu'elle soit asyndétique ou non.

Un autre constat que l'on peut faire est que les verbes recteurs de SA en ancien français sont les mêmes que les recteurs faibles du français moderne (voir Blanche-Benveniste, 1989, Andersen, 1996, Schneider, 2007). Leur fonctionnement grammatical est cependant différent : en ancien français, la négation du verbe recteur n'est pas restreinte aux verbes d'empêchement (type $i l$ n'empêche), elle apparaît avec un verbe de croyance (cuidier) ; l'emploi des temps et des personnes est plus varié puisque la SA peut suivre des verbes d'opinion au passé et à la $3^{\text {ème }}$ personne (et non à la $1^{\text {ère }}$ personne du présent de l'indicatif comme en FM).

Comme nous l'avions signalé ci-dessus, ce phénomène est considéré comme typique de l'ancien français et disparu par la suite : «Le plus souvent il s'agit de « complétives » juxtaposées, mais cette construction ne semble pas dépasser le milieu du XVe siècle » (Marchello-Nizia 1979, $1: 428$ ); "Les consécutives non introduites ne sont pas fréquentes, et semblent limitées au tout début du XIVe siècle et aux textes les plus conservateurs » (Marchello-Nizia 1979, $1: 428$ ) ; "Cette construction [effacement du relatif] ne se rencontre plus après le XIIIe siècle. » (Marchello-Nizia 1998 : 314). Cependant, comme nous avons pu le voir, il semble qu'on puisse en trouver des échos en français moderne, du moins à l'oral : «Quant à la possible omission de que dans une position où le français standard l'impose, c'est un phénomène si peu décrit en France que beaucoup le donnent comme typique du Québec, où il est mieux décrit. Pourtant, il est bien attesté en français de France » (Chaurand $1999: 616)$; «Ce phénomène [complétive en parataxe] est encore possible à l'oral en FNS [français non standard] » (Marchello-Nizia 1999 : 69) ; « Lorsque les deux membres de la phrases sont séparés par une subordonnée, le français moderne a conservé la possibilité d'effacer le que [en corrélation avec tant, tel, si] » (Marchello-Nizia 1998 : 314).

En effet, nous avons déjà parlé du fait que les SA complétives de l'ancien français peuvent être rapprochées des recteurs faibles (ou verbes parenthétiques) en français moderne. De même, on pourrait envisager un parallèle entre les SA relatives de l'ancien français et certaines structures en il y a du français moderne pour lesquelles les auteurs s'interrogent sur la question de savoir si l'on doit considérer une structure du type il y a $\mathrm{N}$ il $\mathrm{P}$ comme une variante de la structure à relative de type il y a $\mathrm{N}$ qui $\mathrm{P}$ (voir entre autres Willems à par.; Berrendonner à par.). On peut également observer que la construction paratactique du système hypothétique à verbe en tête existe toujours en français (voir aussi à ce sujet Béguelin \& Corminboeuf 2005).

Toutefois, s'il semble qu'on puisse trouver des caractéristiques communes à travers le temps qui amènent à penser la parataxe en termes de continuité, il existe cependant certaines différences. En effet, bien qu'il semble qu'on puisse établir un lien de ressemblance avec certaines constructions du français moderne, toutes les grammaires donnent la parataxe comme se raréfiant à partir de la fin de l'ancien français, ce qui peut faire penser que l'évolution de la parataxe est liée au type de langue, la langue française ayant changé de 'type' à l'époque du moyen français 9 .

Par ailleurs, en ancien français, on a pu observer une différence entre les textes en prose et les textes en vers, la parataxe étant plus présente dans les textes en vers. Cette caractéristique amènerait à penser que la présence de la parataxe est liée au genre de texte, puisque même au sein des textes en vers, elle apparaît davantage dans les chansons de geste. Il peut paraître normal qu'il y ait davantage de parataxe dans les textes en vers que dans la prose. D'une part, la contrainte métrique peut amener à choisir la parataxe pour respecter le nombre de syllabes. D'autre part, C. Marchello-Nizia $(1978)^{10}$ a montré que le rythme, soit à la limite de vers, soit à l'hémistiche, sert à délimiter les propositions, et donc facilite la parataxe, tandis que la prose a davantage recours aux joncteurs pour délimiter les propositions.

Mais cette différence entre la prose et les vers, plutôt qu'une différence purement de style littéraire, peut également tenir de la différence entre l'oral et l'écrit, par rapport au type de marquage. En effet, les textes en vers étant habituellement récités à l'oral, l'orateur pouvait le réciter avec l'intonation, la prosodie aidant. Le texte pourrait donc comporter des marques d'oralité. La forme versifiée peut donc être 
rapprochée de la forme orale par la présence du rythme, de la prosodie, donc du non-segmental, qui jouent le même rôle que l'intonation, le supra-segmental, à l'oral.

De plus, en français moderne, on remarque également que les cas de parataxe sont, le plus souvent, extraits de corpus oraux. Ainsi, ce pourrait être ce rapport à l'oralité qui rendrait comparable le phénomène des SA en ancien français et en français moderne (voir Gadet 2006).

Deux hypothèses se profilent alors :

Une première hypothèse serait de considérer que la parataxe est liée au type de langue, et dans ce cas, la 'réapparition' de la parataxe en français moderne laisserait penser qu'un nouveau changement typologique est en train de se produire en français, et il faudrait alors observer d'autres phénomènes de changement pouvant confirmer ou infirmer cette hypothèse.

Une autre hypothèse consisterait à voir la parataxe comme étant liée à une différence entre l'oral et l'écrit. On a vu que la parataxe est plus présente à l'oral en français moderne, et dans les textes en vers en ancien français. De plus, les études sur le français oral sont relativement récentes, on ne peut donc pas savoir si la parataxe a 'réapparu', ou bien si elle a toujours coexisté avec les formes introduites, à l'oral, sans qu'on en ait de traces. Si l'on considère la parataxe comme une forme d'expression plus synthétique que la version introduite, alors l'oral serait plus synthétique que l'écrit, et là encore il faudrait étudier d'autres phénomènes pour confirmer ou infirmer cette hypothèse.

Il faudrait donc se demander si ce phénomène de SA constitue une marque de changement typologique, et, si oui, établir si le changement typologique du français s'est fait dans le temps, et s'il y a alors à nouveau un changement typologique du français de nos jours, ou si la différence entre oral et écrit est telle qu'il y a une tendance typologique différente entre les deux.

L'approche diachronique des SA permet donc de mesurer, dans l'histoire du français, la part de changement et de stabilité, dans l'intrication de faits liés à la syntaxe, à la communication (oral vs écrit) et à la typologie textuelle.

\section{Références bibliographiques}

Andersen H. L. (1996). Verbes parenthétiques comme marqueurs discursifs. In Muller C. (éd.), Dépendance et intégration syntaxique, Tübingen : Niemeyer, 307-315.

Béguelin M.-J. \& Gilles Corminboeuf, (2005). De la question à l'hypothèse : modalités d'un phénomène de coalescence. In Rossari C., A. Beaulieu-Masson, C. Cojocariu, A. Razgouliaeva, (éd.), Les États de la question, Québec : Nota bene, 67-89.

Berrendonner A. (à par.). L’alternance que / \#. Subordination sans marqueur ou structure périodique ? In Van Raemdonck D. (éd.), Actes du Colloque Modèles syntaxiques, Bruxelles, 6-8 mars 2003.

Bertin A. (1997). L'expression de la cause en ancien français. Genève : Droz.

Blanche-Benveniste C. (1989). Constructions verbales "en incise" et rection faible des verbes. Recherches sur le français parlé, 9, 53-73.

Böhme-Eckert G. (2004). De l'ancien français au français moderne : l'évolution vers un type «à part » à l'époque du moyen français. Langue française, 141, 56-68.

Bonnard H., Régnier C. (1997). Petite Grammaire de l'ancien français. Paris : Magnard.

Buridant C. (2000). Grammaire nouvelle de l'ancien français. Paris : SEDES.

Chaurand J. (éd.) (1999). Nouvelle histoire de la langue française. Paris : Seuil.

Foulet L. (1928). Petite Syntaxe de l'ancien français. Paris : Honoré Champion, 1998.

Gadet F (2006). Hier comme aujourd'hui. In Guillot C., Heiden S., Prévost S. (éd.), A la quête du sens, Lyon : ENS Éditions, 191-198. 
Glikman J. (à par. a). Peut-on établir des critères formels de reconnaissance de la parataxe : l'apport de l'ancien français. Colloque international La Parataxe, Neuchâtel, Suisse, 12-15 février 2007.

Glikman J. (à par. b). Les complétives non introduites en ancien français. Colloque international Diachro-3, Paris, 2022 septembre 2006, éditions Peter Lang.

Marchello-Nizia C. (1978). Ponctuation et 'unités de lecture' dans les manuscrits médiévaux, ou je ponctue, tu lis, il théorise. Langue Française, 40, 32-44.

Marchello-Nizia C. (1979). Histoire de la langue française au XIVe et XVe siècle. Paris : Nathan, 1997.

Marchello-Nizia C. (1993). Parataxe et syntaxe du vers en ancien français. In Perrin I. (éd.), Approches énonciatives de l'énoncé complexe, Louvain-Paris : Peeters, 63-71.

Marchello-Nizia C., Picoche J. (1998). Histoire de la langue française. Paris : Nathan.

Marchello-Nizia C. (1999). Le français en diachronie : douze siècles d'évolution. Paris : Ophrys.

Ménard P. (1988). Syntaxe de l'ancien français. Bordeaux : Bière, 1994.

Schneider S. (2007). Les clauses parenthétiques réduites en français, italien, et espagnol. Une analyse pragmatique fondée sur des corpus de la langue parlée. In David Trotter (éd.). Actes du XXIVe Congrès International de Linguistique et de Philologie Romanes. Aberystwyth, 2-5 août 2004. Volume 3. Tübingen : Niemeyer, 423-432.

Soutet O. (1990). La concession en français. Genève : Droz.

Weill I. (2002). Tant, étude d'un marqueur de transfert dans les textes épiques du Moyen Français. LINX, numéro spécial, 2002, 405-412.

Willems Dominique \& Machteld Meulleman (à par.). «Il y a des gens ils viennent acheter des aspirines pour faire de l'eau gazeuse ». Sur les raisons d'être des structures parataxiques en il y a. In Béguelin, M.J., Avanzi, M. et Corminboeuf, G. Actes du colloque international La Parataxe, Neuchâtel, Suisse, 12-15 février 2007.

Adam de la Halle, Le Jeu de la Feuillée, Paris : Lettres gothiques.

Adenet le roi, Berte aus grans piés. A. Henry (éd.), Bruxelles : P U de Bruxelles, 1963.

Antoine de la Sale, Jehan de Saintré. J. Blanchard (éd.), Paris : Lettres Gothiques, Le Livre de Poche, 1995, 1455.

Chrétien de Troyes, Erec et Enide. M. Roques (éd.), Paris : Champion, 1981.

Documents linguistiques de la Belgique Romane. Publiés par J. Monfrin, I. Hainaut, P. Ruelle (éd.), Paris : Edition du CNRS 1984.

Documents linguistiques de la France (série française), publiés par J. Monfrin, III Aube, Seine-et-Marne, Yonne, D. Coq (éd.), Paris : Edition du CNRS 1988.

François Villon, Euvres. A. Longnon (éd.), $4^{\mathrm{e}}$ éd. revue par L. Foulet, Paris : Champion, 1992.

Histoire de la reine Berthe et du roy pepin. P. Tylus (éd.), Genève : Droz, 2001.

Jehan Bodel, Le jeu de Saint Nicolas. A. Henri (éd.), Genève : Droz, 1981.

La chanson de Roland. C. Segre (éd.), Genève : Droz, 2003.

La Farce de Maitre Pierre Pathelin, J. Dufournet (éd.), Paris : Flammarion, 1986.

La Mort le Roi Artu. J. Frappier (éd.), Genève : Droz, 1996.

La Passion du Palatinus. G. Franck (éd.), Paris : Champion, 1972.

La vie de Saint Alexis, M. Perugi (éd.), Genève : Droz, 2000.

La vie de Sainte Marie l'Egyptienne, versions en ancien et en moyen français. P. F. Dembowski (éd.), Genève : Droz, 1977.

Le Roman de Renart, M. Roques (éd.), Paris : Champion, 2007.

Le Roman de Renart, Branche I, H. Rey-Flaud et A. Eskénazi (trad.), Paris : Champion, 1982.

Les Rédactions en vers du Couronnement de Louis, Y. G. Lepage (éd.), Paris-Genève : Droz, 1978. 
Les séquences de Sainte Eulalie. Roger Berger, Annette Brasseur (éds), Paris : Droz, 2004.

Robert de Clari, La conquête de Constantinople. J. Dufournet (éd.), Paris : Champion, 2004.

Rutebeuf, Euvres complètes. M. Zink (éd.), Paris : Classiques Garnier, 2001.

Base de français médiéval (BFM) : http://bfm.ens-lsh.fr/

Base textuelle Frantext pour l'agrégation : $\underline{\text { http://www.frantext.fr/agregation.htm }}$

Concordanciers sur le site de l'université Rennes 2 : http://www.uhb.fr/alc/medieval/actua.htm

\footnotetext{
${ }^{1}$ Je tiens ici à remercier Annie Bertin, Mathieu Avanzi et Christophe Benzitoun qui ont bien voulu relire une version antérieure de cet article et m'ont fourni de précieux conseils et remarques, ainsi que les relecteurs du comité pour leurs observations.

${ }^{2}$ Les traductions, littérales, données ici pour faciliter la compréhension du lecteur non spécialiste, ont été établies à l'aide du glossaire de l'édition de référence et de la traduction de H. Rey-Flaud et A. Eskénazi. Les éléments restitués sont placés entre crochets.

${ }^{3}$ Je crois, tu sais, il n'empêche...

${ }^{4}$ Nous ne tenons pas compte dans notre corpus des cas problématiques afin d'éviter de fausser l'analyse.

${ }^{5}$ Voir ci-dessus en 1.2. le développement sur (35) et (36).

${ }^{6}$ Voir, entre autres, vers $1716-18$ pour un équivalent introduit.

${ }^{7}$ Voir par exemple vers 161-63 pour un équivalent introduit.

${ }^{8}$ Voir les vers 616,628 et 1584 avec que.

${ }^{9}$ Voir par exemple G. Böhme-Eckert 2004 à ce sujet.

${ }^{10}$ Voir aussi dans Bertin 1997 : 152.
} 